\title{
INCLUSIVE AND SEMI-INCLUSIVE PION PRODUCTION IN pp COLLISIONS AT 102 AND $400 \mathrm{GeV} / c^{\star}$
}

\author{
C. BROMBERG * ${ }^{\star}$, T. FERBEL and P. SLATTERY \\ University of Rochester, Rochester, NY 14627
}

\author{
A.A. SEIDL and J.C. VANDER VELDE \\ University of Michigan, Ann Arbor, Mich. 48104
}

Received 17 February 1976

\begin{abstract}
We present a comparative study of inclusive and semi-inclusive pion production in pp collisions at 102 and $400 \mathrm{GeV} / c$. In particular, we examine the correlation between transverse and longitudinal momentum variables and the energy dependence of invariant cross sections.
\end{abstract}

\section{Introduction}

In this paper we present a comparative study of inclusive and semi-inclusive pion production in proton-proton collisions at 102 and $400 \mathrm{GeV} / c$, and specifically investigate the validity of various scaling hypotheses which have been proposed for particle production in high-energy collisions $\dagger$. The data are from an analysis of inelastic events $(\sim 3000$ events at $102 \mathrm{GeV} / c$ and $\sim 2200$ events at $400 \mathrm{GeV} / c)$ obtained in exposures of the FNAL 30 -inch bubble chamber to proton beams at the highest available accelerator momenta ${ }^{*}$.

A difficulty in the analysis of bubble chamber data at high energies is the lack of particle identification for tracks of momenta in excess od $1.2 \mathrm{GeV} / c$; below this momentum ionization estimates in the bubble chamber can be used to separate protons from pions. To correct for proton contamination of the $\pi^{+}$data above $1.2 \mathrm{GeV} / c$ lab momentum, we have assumed that the observed cross sections for inclusive and semi-inclusive proton production, at momenta below $1.2 \mathrm{GeV} / c$, extra polate smoothly to higher momenta. The inclusive proton spectrum has been shown previously [3] to be almost independent of the scaling variable $x=2 p_{\ell}^{*} / \sqrt{s}$ for $x$

\footnotetext{
* Research supported by the US Energy Research and Development Adminstration.

$\star \star$ Present address: Calif. Institute of Technology, Pasadena; Calif. 91125.

$\uparrow$ For a summary of the general features of multiparticle production at high energies, see the reviews given in ref. [1].

Preliminary results from the $102 \mathrm{GeV} / c$ exposure may be found in ref. [2].
} 
values in the range $0.8 \gtrsim|x| \gtrsim 0.5$ ( $p_{0}^{*}$ is the c.m. longitudinal momentum and $\sqrt{s}$ is the c.m. energy in the collision). In calculating corrections to the $\pi^{+}$distributions we have assumed that the inclusive cross section for protons in the region $|x|<0.5$ remains unchanged from that observed for $0.5<|x|<0.8$. Corrections for proton contamination of the $\pi^{+}$spectra were calculated using a Monte-Carlo program, and the results are displayed along with the uncorrected $\pi^{+}$spectra whenever these corrections are significant.

In the text we refer to the positive spectra as $\pi^{+}$and negative spectra as $\pi^{-}$. However, we estimate ${ }^{*}$ that on the average there exists approximately $1 \% \mathrm{e}^{-}, 7 \% \mathrm{~K}^{-}$ and $2 \% \overline{\mathrm{p}}$ contamination in the displayed negative spectra, and approximately $1 \% \mathrm{e}^{+}$ and $10 \% \mathrm{~K}^{+}$contamination in the positive spectra (in addition to the calculated proton contamination).

\section{Inclusive pion production}

The inclusive scaling hypotheses of Wilson [5], of Feynman [6] and of Benecke et al. [7] predict that the invariant cross section for single-particle production at fixed values of transverse and longitudinal momentum in the target or projectile rest frame, approaches an energy-independent limit as the colliding energy increases. Expressing the invariant cross section in terms of rapidity in the target frame, $y_{\text {lab }}=\frac{1}{2} \ln \left[\left(E+p_{\ell}\right) /\left(E-p_{\ell}\right)\right]$, and the transverse momentum $p_{\mathrm{T}}$ of the produced particle, the essence of these scaling hypotheses can be written as follows:

$$
\frac{E \mathrm{~d} \sigma}{\mathrm{d}^{3} p}=f\left(y_{\mathrm{lab}}, p_{\mathrm{T}}, s\right) \stackrel{s \rightarrow \infty}{\longrightarrow} f\left(y_{\mathrm{lab}}, p_{\mathrm{T}}\right),
$$

where the region $p_{\ell}^{*} \sim 0$ (i.e., $y^{*} \sim 0$ ) is explicitly excluded from the predictions. More recently, Mueller [8] was able to show that inclusive scaling follows from a generalized optical theorem combined with a Regge picture of particle production in which the invariant cross section is expected to develop a "plateau" in the central region of rapidity space as $s$ becomes very large:

$$
f\left(y^{*}, p_{\mathrm{T}}\right) \stackrel{s \rightarrow \infty}{\longrightarrow} f\left(p_{\mathrm{T}}\right) .
$$

Using the Mueller-Regge picture, the scaling hypothesis can therefore be extended into the previously excluded region of $p_{Q}^{*} \approx 0$.

We begin our study by displaying in fig. 1 the inclusive production spectra for $\pi^{+}$and $\pi^{-}$at 102 and $400 \mathrm{GeV} / c$. The data at the two beam momenta are presented

\footnotetext{
\# Material in ref. [1] has been used to estimate $\overline{\mathrm{p}}$ and $\mathrm{K}^{ \pm}$background. Kaon contamination was also estimated from data on $\mathrm{K}_{S}^{0}$ production (see ref. [4]). Electron contamination stems from the Dalitz decay mode of $\pi^{0}$ mesons.
} 


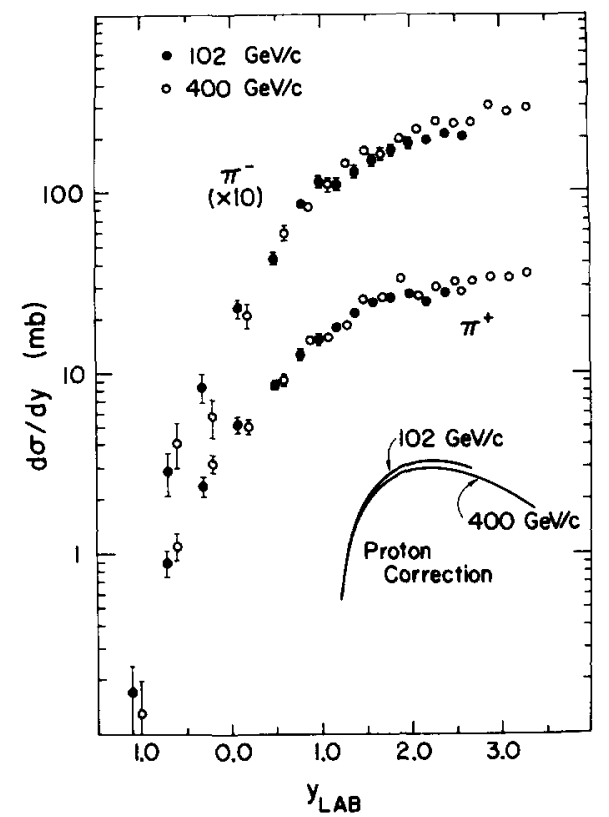

Fig. 1. Single-particle inclusive spectra for $\pi^{--}$and $\pi^{+}$production. The spectra for positive pions have not been corrected for contamination from kaons, electrons or protons. The estimated corrections for proton contamination of the $\pi^{+}$data are sketched in the figure (smooth curves).

in terms of a cross section as a function of $y_{\text {lab }}$ integrated over $p_{\mathrm{T}}$ and azimuth,

$$
\frac{\mathrm{d} \sigma}{\mathrm{d} y_{\mathrm{lab}}}=\pi \int \mathrm{d} p_{\mathrm{T}}^{2} E \frac{\mathrm{d} \sigma}{\mathrm{d}^{3} p}=\int \mathrm{d} p_{\mathrm{T}}^{2} \frac{\mathrm{d} \sigma}{\mathrm{d} y_{\mathrm{lab}} \mathrm{d} p_{\mathrm{T}}^{2}} .
$$

We show only the data for production in the backward hemisphere of the center of mass $\left(y_{\text {lab }}<2.69\right.$ at $102 \mathrm{GeV} / \mathrm{c}$ and $y_{\text {lab }}<3.37$ at $\left.400 \mathrm{GeV} / c\right)$, where background and systematic errors are least severe. Also shown are the corrections that must be applied to the $\pi^{+}$data points to account for proton background. The errors contain statistical as well as systematic uncertainties due to measuring and scanning difficulties. The data are consistent to within $\sim 10 \%$ uncertainty with the scaling of $\pi^{+}$and $\pi^{-}$production in the target fragmentation region for rapidity values of $y_{\text {lab }} \leqslant 1.5$. However, we point out that in a recent detailed study of the energy dependence of the cross section for the fragmentation region $\left(y_{\text {lab }}<0\right)$, we have found a significant decrease in pion production from AGS to NAL energies [9] which indicates that scaling in the fragmentation regime may be approached from above. The approach to scaling throughout the fragmentation region remains an interesting question for probing with higher statistics experiments.

In contrast to the result in the fragmentation region, there is a large growth of the pion cross section in the central region $\left(y_{1 a b} \geq 1.5\right)$. In particular, we find that 
the cross sections at $y^{*}=0$ (i.e., $y_{1 \mathrm{ab}}=2.69$ at $102 \mathrm{GeV} / c$ and $y_{1 \mathrm{ab}}=3.37$ at $400 \mathrm{GeV} / \mathrm{c})$ have increased by $(45 \pm 10) \%$ for the $\pi^{-}$data and $(30 \pm 10) \%$ for the $\pi^{+}$data. Application of Mueller-Regge techniques to pion production at $y^{*}=0$ suggests a rather slow approach to scaling: $\mathrm{d} \sigma(s) /\left.\mathrm{d} y\right|_{y^{*}=0}=a+b s^{-1 / 4}[8,10]$. The 102 and $400 \mathrm{GeV} / c$ data points, corrected for $\mathrm{K}$, e and $\mathrm{p}$ backgrounds, yield the following values for these parameters:

$$
\begin{array}{lcc} 
& a(\mathrm{mb}) & b\left(\mathrm{mb} \cdot \mathrm{GeV}^{1 / 2}\right) \\
\pi^{-} & 46 \pm 6 & -100 \pm 25 \\
\pi^{+} & 44 \pm 6 & -80 \pm 30
\end{array}
$$

The values of $a(\sim 45 \mathrm{mb})$ are about two standard deviations larger than the corresponding values obtained from extrapolations using low-energy experiments [11].

We next investigate the transverse momentum characteristics of $\pi^{-}$production. In fig. 2 the first two moments of the $p_{\mathrm{T}}$ spectra at fixed $y_{\text {lab }}$ are shown; the $n$th moment is given by

$$
\left\langle p_{\mathrm{T}}^{n}\right\rangle=\int \mathrm{d} p_{\mathrm{T}}^{2} p_{\mathrm{T}}^{n} \frac{E \mathrm{~d} \sigma}{\mathrm{d}^{3} p} / \int \mathrm{d} p_{\mathrm{T}}^{2} \frac{E \mathrm{~d} \sigma}{\mathrm{d}^{3} p} .
$$

The integration limits are $0<p_{\mathrm{T}}<1.5 \mathrm{GeV} / c$. We observe that the moments are $s$-independent at fixed $y_{\text {lab }}$. Also, the trend of the data with expanding central rapidity region suggests that $\pi^{-}$production has nearly attained a limiting value of $\left\langle p_{\mathrm{T}}\right\rangle \sim 0.36 \mathrm{GeV} / c$. The second moment would appear to require somewhat higher energies before possibly reaching a saturation value. (The decrease of the moments for $y_{\text {lab }} \leqslant 0$ may be due to kinematic restrictions on $p_{\mathrm{T}}$ for these $y_{\text {lab }}$ values.)

The dependence of the $\pi^{-}$invariant cross section on $p_{\mathrm{T}}$ and $x$ is shown in fig. 3 .
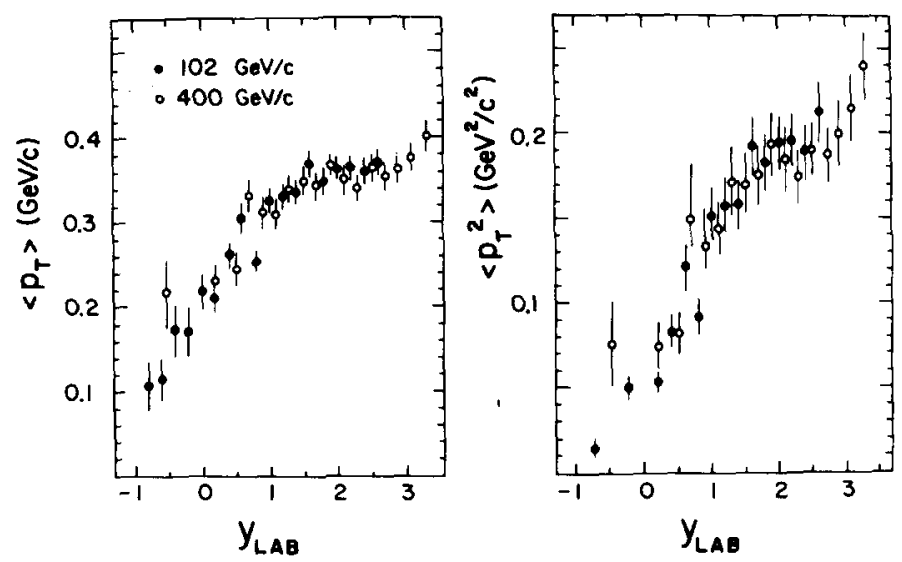

Fig. 2. First two moments (see text) of the $\pi^{-}$transverse momentum spectra as a function of rapidity. 


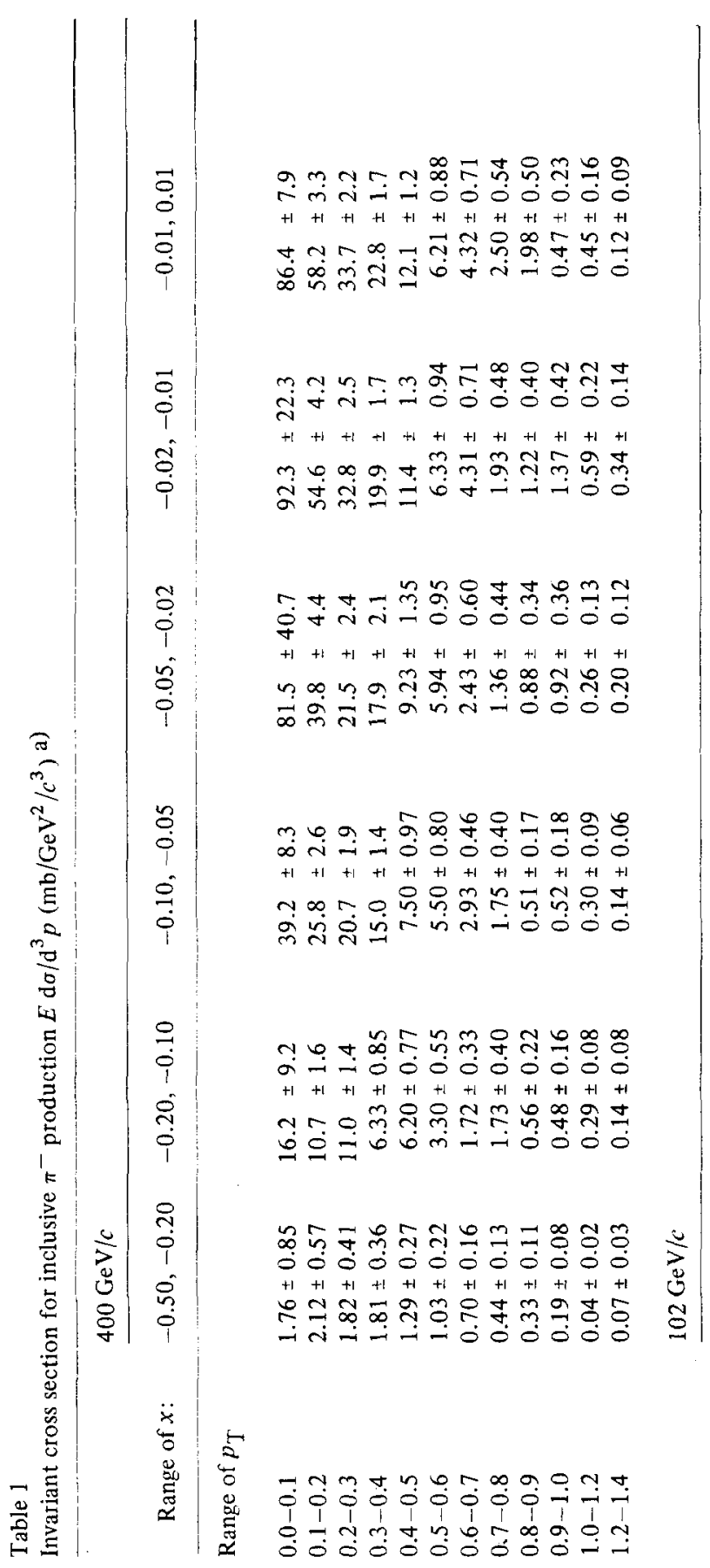

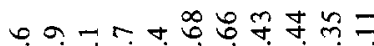
क्ष

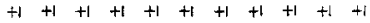
iबं

$\operatorname{nnn\infty }$ $\infty \mathrm{m}$ - $-\dot{0} 000000$ $+1+1+1+1+1+1+1+i+1+1+1$ matr.

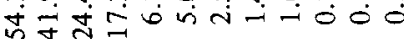

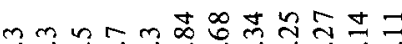
向 $+1+1+1+1+1+1+1+1+1+1+1+1$

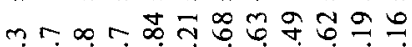

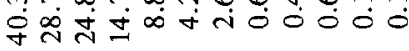

$\infty+r-m B$ ำ $\infty$ ल -

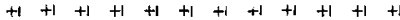

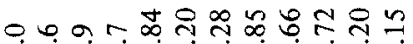

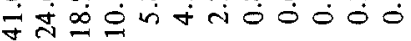

ำกำ

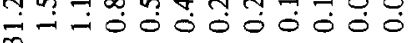
$+1+1+1+i+1+1+1+1+1+1+1+1$ $\because n g$ 苛 m $m$ =

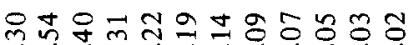
$+1+++1+1+1+1+1+1+1+1+1$

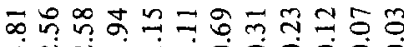
मतन-

ㄱำ 0 $0_{1} 000000$

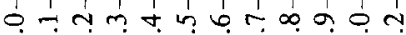




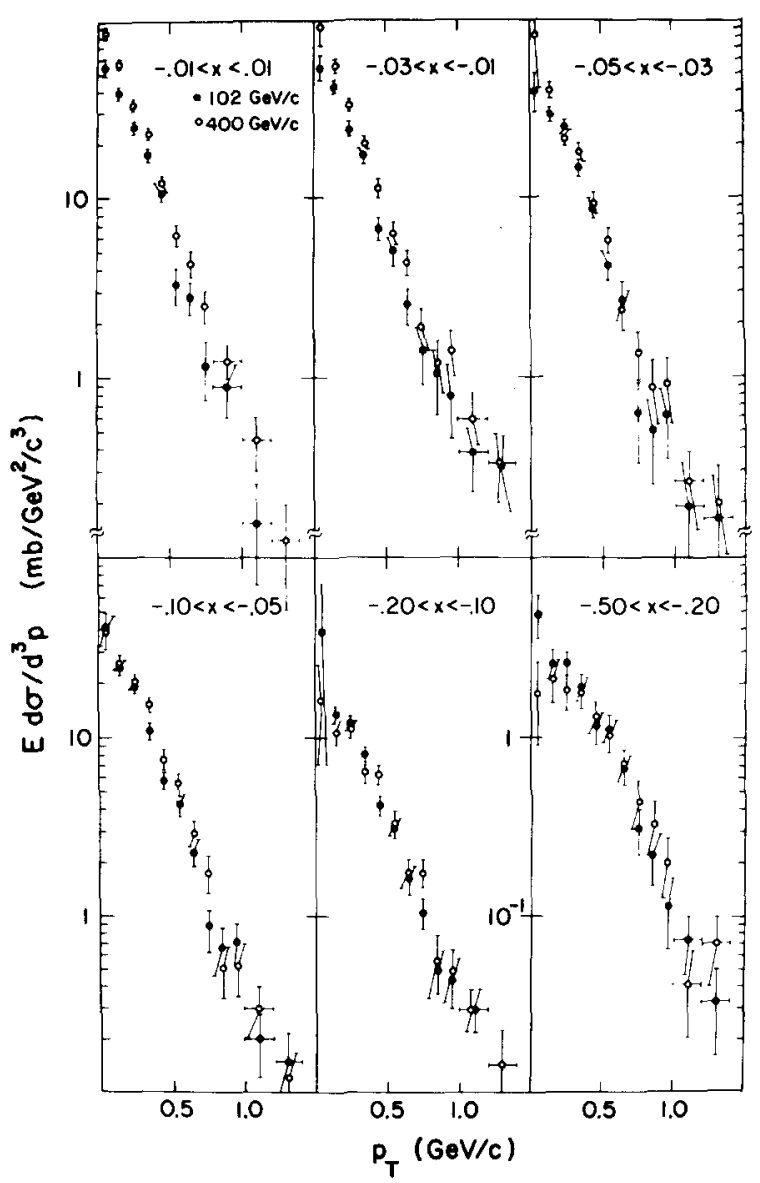

Fig. 3. Single-particle spectra for $\pi^{-}$production. The data have not been corrected for contaminants.

The data are also tabulated in table 1. Similar data have been reported at nearby energies from experiments at FNAL and at the ISR $\dagger$. The scaling violations, noted previously in the rapidity spectra integrated over $p_{\mathrm{T}}$, are also evident here in the growth of the cross sections for $|x| \lesssim 0.03$. In this small- $x$ region the cross section for $p_{\mathrm{T}}$ values between 0.2 and $1.5 \mathrm{GeV} / c$ appears to rise uniformly between 102 and $400 \mathrm{GeV} / c$. Below $p_{\mathrm{T}} \sim 0.2 \mathrm{GeV} / c$, the data exhibit a somewhat more rapid growth with increasing $s$. For the region $|x|>0.05$ the distribution is only weakly energy dependent over the entire range of $p_{\mathrm{T}}$ values; a possible decrease of the cross section with increasing energy is observed at small $p_{\mathrm{T}}$ in the largest $|x|$ region

${ }^{\dagger}$ For excellent compilations of $p_{\mathrm{T}}$ dependences of cross sections, see ref. [12]. 


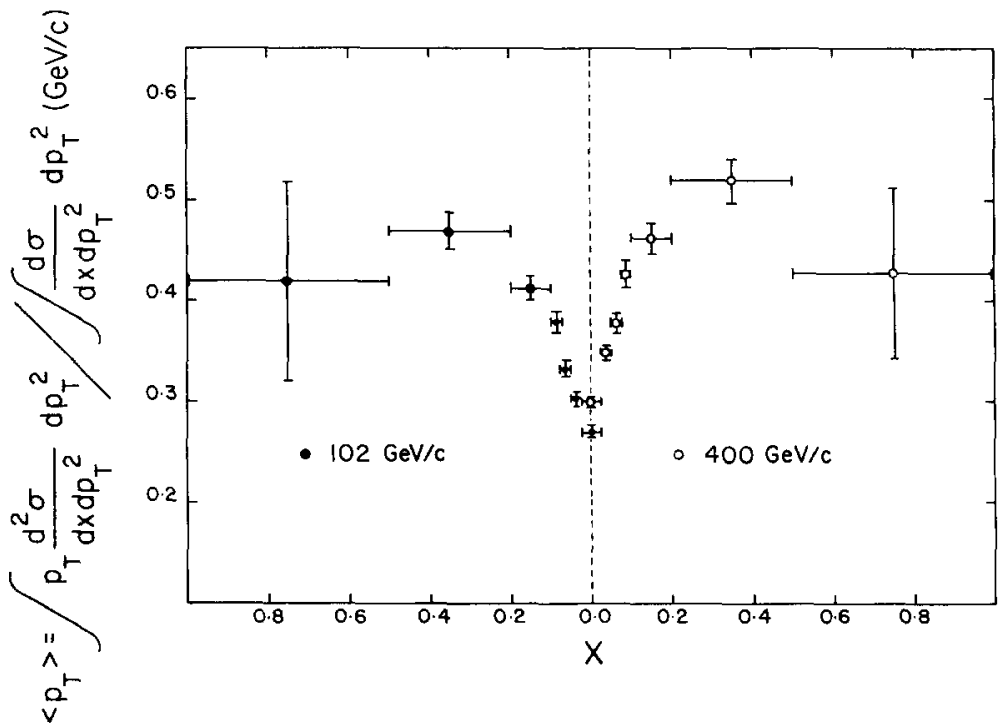

Fig. 4. First moment (see text) of the $\pi^{--}$transverse momentum spectra as a function of the $x$ variable.

shown $(-0.50<x<-0.20)$. As the value of $|x|$ increases, the data appear to exhibit a flattening of the invariant cross section at small $p_{\mathrm{T}}$ (i.e., on the average, higher $p_{\mathrm{T}}$ values appear to be more favored at larger $x$ ). This fact is reflected in the "sea gull" effect [13] observed in fig. 4 where the $\left\langle p_{\mathrm{T}}\right\rangle$ is shown to increase with $|x|$ for $|x|<0.5$. (For clarity the $400 \mathrm{GeV} / c$ data have been plotted after reflection about $x=0$.) A comparison of the behavior of $\left\langle p_{\mathrm{T}}\right\rangle$ (figs. 2 and 4 ) suggests that the invariant cross section is described more simply using the rapidity variable rather than $x$.

Summarizing our findings for inclusive pion production, we observe single-particle spectra for $\pi^{+}$and $\pi^{-}$which are consistent with scaling in the fragmentation region, but exhibit sizeable violations of scaling $(\sim 30 \%)$ in the central region. The $\left\langle p_{\mathrm{T}}\right\rangle$ for $\pi^{-}$seems to saturate near $\left\langle p_{\mathrm{T}}\right\rangle \sim 0.36 \mathrm{GeV} / c$ for production in the central region of rapidity. In the small- $x$ region, particularly for $|x|<0.03$, scaling violations for $\pi^{-}$production are observed throughout our $p_{\mathrm{T}}$ range $(0-1.5 \mathrm{GeV} / c)$ with the violations being most pronounced for the rather low $p_{\mathrm{T}}$ data of $p_{\mathrm{T}} \leqslant 0.2 \mathrm{GeV} / c^{\text {}}$ 米

\section{Semi-inclusive pion production}

The asymptotic scaling of inclusive production has many interesting consequences. In particular, Koba, Nielsen and Olesen (KNO) have shown that the assumption

\# These results are consistent with the latest precision measurements of inclusive pion production at the ISR reported by the British-Scandinavian Collaboration [14]. 
of inclusive scaling leads to a scaling of the multiplicity distribution of the kind [15]

$$
\sigma_{n} \stackrel{s \rightarrow \infty}{\longrightarrow} \frac{1}{\langle n\rangle} \psi\left(\frac{n}{\langle n\rangle}\right) .
$$

Here $n$ refers to the inelastic charged-particle multiplicity and $\psi$ is the scaling function. Recent tests of this hypothesis (KNO-1) have shown [16] that the multiplicity data at FNAL energies are in good agreement with its predictions; although this may seem puzzling when one considers the observed scaling violations noted in the previous section, it has been pointed out by Weingarten that multiplicity scaling does not require that Feynman scaling hold to high accuracy [17]. Extending their work, KNO have proposed semi-inclusive single-particle densities should also scale in a similar manner (KNO-2) [18]

$$
\rho_{n}\left(y, p_{\mathrm{T}}, s\right)=\frac{1}{\sigma_{n}} E \frac{\mathrm{d} \sigma_{n}}{\mathrm{~d}^{3} p} \stackrel{s \rightarrow \infty}{\longrightarrow} \phi\left(y, p_{\mathrm{T}}, \frac{n}{\langle n\rangle}\right) .
$$

The semi-inclusive densities integrated over $p_{\mathrm{T}}$ for $\pi^{-}$and $\pi^{+}$production at 102 and $400 \mathrm{GeV} / c$ are shown in figs. 5 and 6 . As for the inclusive spectra, we show only the data from the backward hemisphere of the center of mass (for economy
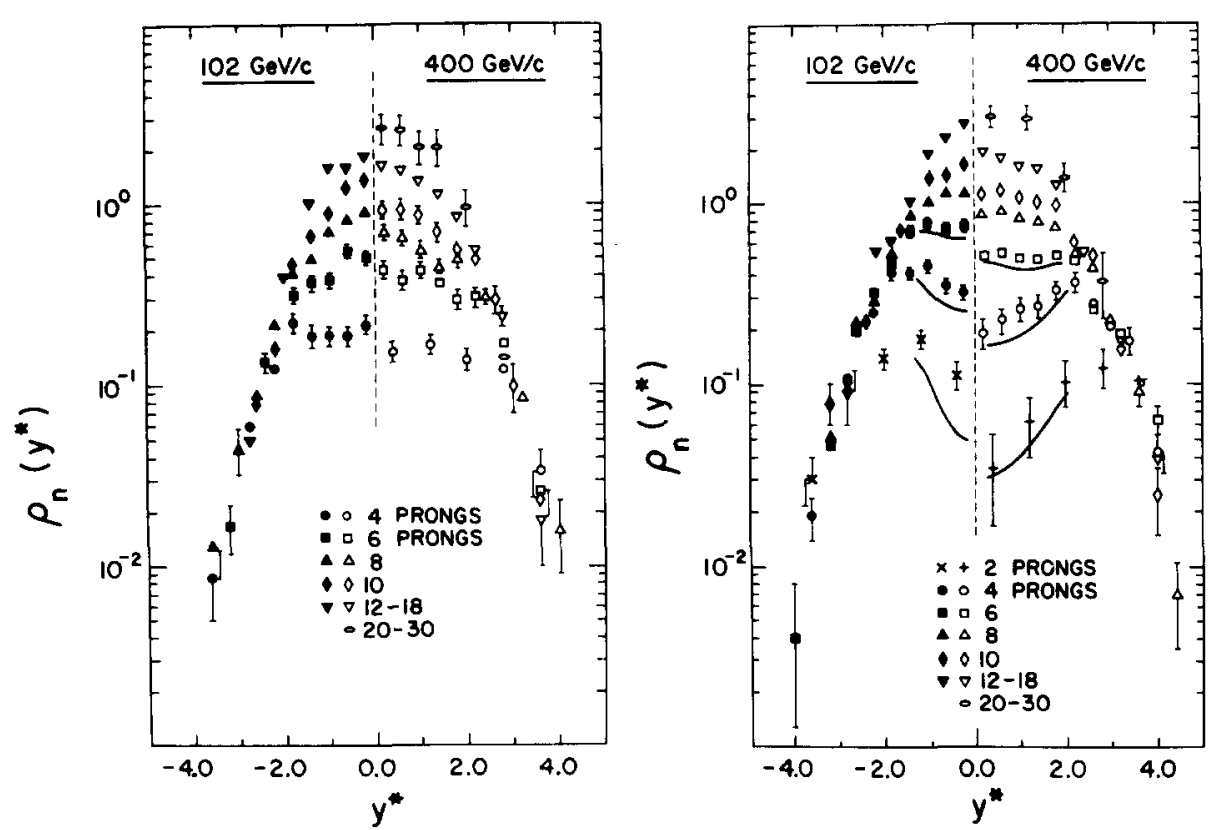

Fig. 5. Semi-inclusive single-particle densities for $\pi^{-}$and $\pi^{+}$production, in (a) and (b), respectively. The data at $400 \mathrm{GeV} / c$ are shown reflected about $y^{*}=0$. The curves shown in (b) for the low multiplicity results $(n=2,4,6)$ represent estimates of the $\pi^{+}$data corrected for proton background. The higher multiplicity data have not been corrected for any contaminants. 


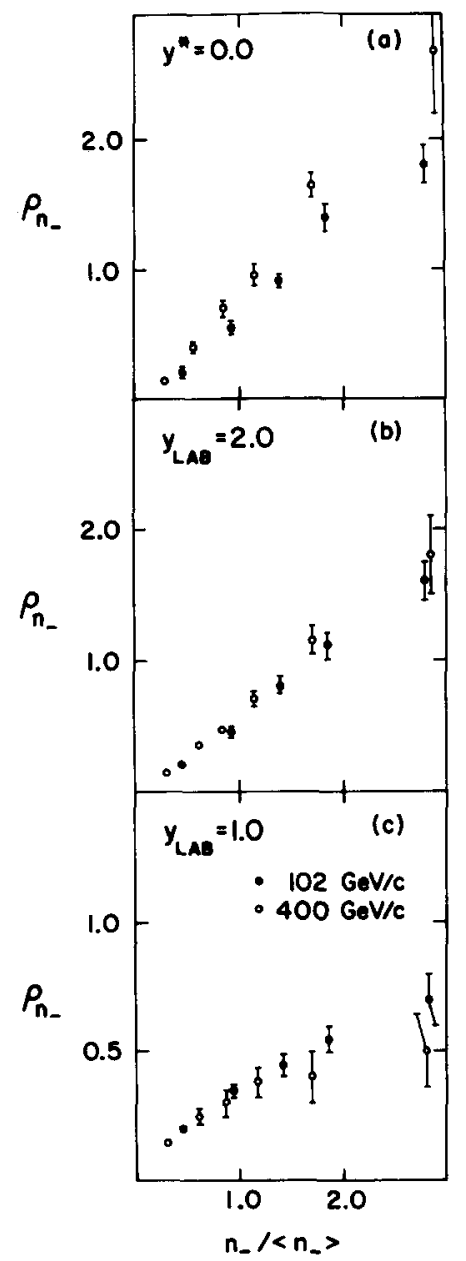

Fig. 6. Semi-inclusive single-particle densities as a function of the KNO scaling variable $n_{-} /\left\langle n_{-}\right\rangle$.

of presentation the $400 \mathrm{GeV} / c$ data have been plotted after reflection about $y^{*}=0$ ) The curves shown under the low-multiplicity results in fig. $6(n=2,4,6)$ represent $\pi^{+}$data corrected for proton contamination (corrections for the higher multiplicities have not been calculated). For the highest multiplicities the cross sections have been summed over the indicated range of $n$.

We note that while the semi-inclusive densities at $y^{*}=0$ often differ by orders of magnitude, at large $\left|y^{*}\right|$ the density appears to be only weakly dependent on the charge multiplicity $n$. Although the individual spectra in fig. 5 do not show evidence for the presence of a central plateau (save for perhaps the $n=4 \pi^{-}$data), there is apparently a general flattening of the $\pi^{--}$data as the beam momentum in- 
Table 2

Mean transverse momenta for $\pi^{-}$mesons

\begin{tabular}{|c|c|c|c|c|c|}
\hline \multirow{3}{*}{$\begin{array}{l}\text { Incident } \\
\text { momentum } \\
(\mathrm{GeV} / \mathrm{c})\end{array}$} & \multicolumn{5}{|l|}{$\left\langle p_{\mathrm{T}}\right\rangle(\mathrm{GeV} / c)^{\mathrm{a})}$} \\
\hline & \multicolumn{5}{|c|}{ Prong multiplicity } \\
\hline & $4-6$ & $8-10$ & $12-18$ & $20-30$ & all \\
\hline 102 & $0.351 \pm 0.007$ & $0.345 \pm 0.007$ & $0.314 \pm 0.015$ & & $0.341 \pm 0.005$ \\
\hline 400 & $0.365 \pm 0.010$ & $0.357 \pm 0.007$ & $0.345 \pm 0.006$ & $0.357 \pm 0.020$ & $0.352 \pm 0.005$ \\
\hline
\end{tabular}

a) These results have not been corrected for $\mathrm{K}^{-}, \mathrm{e}^{-}$or $\overline{\mathrm{p}}$ contaminants.

creases from 102 to $400 \mathrm{GeV} / c$. The data for $\pi^{+}$production, particularly in the low multiplicities, exhibit enhancements at large $\left|y^{*}\right|$ which are somewhat more pronounced in the corrected spectra (curves in fig. $5 \mathrm{~b}$ ). These peaks can be attributed to the fragmentation of incident protons which produce an excess of positive pions at large $\left|y^{*}\right|$.

Using the $\pi^{-}$data shown in fig. 5a, we have selected three representative rapidity values, $y^{*}=0, y_{1 \mathrm{lab}}=1$ and $y_{1 \mathrm{ab}}=2$, at which to test the validity of the KNO-2 hypothesis. For $\pi^{-}$production, the hypothesis can be written as follows:

$$
\rho_{n_{-}}(y)=\phi\left(y, \frac{n_{-}}{\left\langle n_{-}\right\rangle}\right) \text {, }
$$

where $n_{-}=\frac{1}{2}(n-2)$ is the number of negative particles in each event, $\rho_{n_{-}}$is the particle density function integrated over $p_{\mathrm{T}}$, and $\phi$ is the postulated scaling function. The data in fig. 6 a clearly indicate that no single function of $n_{-}\left\langle\left\langle n_{-}\right\rangle\right.$describes the energy dependence of $\rho_{n_{-}}$at $y^{*}=0$. However, the data in figs. $6 \mathrm{~b}, \mathrm{c}$, for lab rapidities $y_{\text {lab }}=1$ and $y_{1 \mathrm{ab}}=2$, agree more closely with a unique $n_{-}\left\langle n_{-}\right\rangle$dependence. Observations similar to these have been noted in a recent study wherein semi-inclusive $\pi^{--}$production at AGS energies was compared with the FNAL data at $205 \mathrm{GeV} / \mathrm{c}^{\dagger}$.

Thus, we find that in the fragmentation region of rapidity $\left(y_{1 \mathrm{ab}} \leqslant 1.5\right.$ ), where the data are in reasonable agreement with the inclusive scaling hypothesis, the KNO-2 semi-inclusive scaling hypothesis also seems to be valid. Conversely, in the central rapidity region $\left(y^{*} \sim 0\right)$ where the inclusive scaling hypothesis is violated, KNO-2 is also violated ${ }^{\ddagger}$.

$\dagger_{\text {It }}$ is worthwhile to point out that in a more detailed investigation of $\mathrm{KNO}-2$ scaling for momenta between 6 and $400 \mathrm{GeV} / c$ we have found a small but significant systematic disagreement between the data and the scaling prediction in the target fragmentation region (Schindler et al. [19]).

₹ This result is not very surprising because $\mathrm{d} \sigma / \mathrm{d} y$ is related to the $\rho_{n}$ functions and to the KNO-1 scaling function $\psi$ 'ia $\mathrm{d} \sigma / \mathrm{d} y=\int_{0}^{\infty} \mathrm{d} z \rho_{z}(y, z) \psi(z)$, where $z=n_{-} /\left\langle n \_;\right.$; and since $\psi(z)$ is relatively energy independent and do/d $y$ does not scale, particularly in the central 
As a final semi-inclusive characteristic, we display in table 2 the $\left\langle p_{\mathrm{T}}\right\rangle$ values, averaged over rapidity, in the inclusive and semi-inclusive $\pi^{-}$data samples. The slight increase in $\left\langle p_{\mathrm{T}}\right\rangle$ between the two energies is presumably due to additional contributions from central production $\left(\left\langle p_{\mathrm{T}}\right\rangle \sim 0.36 \mathrm{GeV} / c\right)$ in the $400 \mathrm{GeV} / c$ data (c.f. fig. 2). Within our experimental errors, $\left\langle p_{\mathrm{T}}\right\rangle$ displays little or no multiplicity dependence at both energies .

We thank D. Chaney and R. Schindler for help in the early stages of the analysis. We also thank D. Weingarten for a critical reading of the manuscript and for helpful discussions.

\section{References}

[1] J. Whitmore, Phys. Reports 10 (1974) 273;

H. Bøggild and T. Ferbel, Ann. Rev. Nucl. Sci. 24 (1974) 451;

L. Foa, J. de Phys. C1 (1973) 317.

[2] C. Bromberg et al., Phys. Rev. D9 (1974) 1864

[3] J.W. Chapman et al., Phys. Rev. Letters 32 (1974) 257.

[4] J.W. Chapman et al., Phys. Letters 47B (1973) 465.

[5] K. Wilson, Acta Phys. Austriaca 17 (1963) 37.

[6] R.P. Feynman, High-energy collisions, ed. C.N. Yang et al., (Gordon and Breach, New York, 1970) p. 237; Phys. Rev. Letters 23 (1969) 1415.

[7] J. Benecke, T.T. Chou, C.N. Yang and F. Yen, Phys. Rev. 188 (1969) 2159.

[8] A. Mueller, Phys. Rev. D2 (1970) 2963.

[9] R. Schindler et al., Phys. Rev. Letters 33 (1974) 862.

[10] H. Abarbanel, Phys. Rev. D3 (1971) 2227.

[11] T. Ferbel, Phys. Rev. Letters 29 (1972) 448.

[12] P. Capiluppi et al., Nucl. Phys. B70 (1974) 1;

J. Cronin, Proc. Int. School of subnuclear physics, Erice, 1975, ed. A. Zichichi.

[13] M. Baradin-Otwinowska et al., Proc. Sienna Conf. on elementary particles (1963) vol. 1, p. 628 ;

S. Stone et al., Phys. Rev. D5 (1972) 1621.

[14] B. Duff, presentation at Palermo Conf., 1975.

[15] Z. Koba, H.B. Nielsen and P. Olesen, Nucl. Phys. B40 (1972) 317.

[16] P. Slattery, Phys. Rev. Letters 29 (1972) 1674;

P. Olesen, Phys. Letters B41 (1972) 602.

[17] D. Weingarten, Nucl. Phys. B70 (1974) 501.

[18] Z. Koba, H.B. Nielsen and P. Olesen, Phys. Letters 38B (1972) 25.

[19] Z. Ming Ma et al., Phys. Rev. Letters 31 (1973) 1320;

R. Schindler et al., Bull. Am. Phys. Soc. 19 (1974) 467.

[20] T. Ferbel, SLAC Summer Institute, 1974. ed. M. Zipf, SLAC report 179.

region, it follows that the $\rho_{n}$ functions will exhibit a variation with incident momentum, especially in the regime where $\mathrm{d} \sigma / \mathrm{d} y$ does not scale.

* See, however, the data presented in ref. [20]. 